Témoigner Témoigner. Entre histoire et mémoire

Getuigen Revue pluridisciplinaire de la Fondation Auschwitz

$122 \mid 2016$

Révisionisme et négationisme

\title{
Enric Marco, c'est moi : El impostor de Javier Cercas
}

Enric Marco, that's me: El impostor by Javier Cercas

Enric Marco, dat ben ik: El impostor van Javier Cercas

\section{Luc Rasson}

\section{(2) OpenEdition}

Journals

Édition électronique

URL : https://journals.openedition.org/temoigner/4414

DOI : $10.4000 /$ temoigner.4414

ISSN : 2506-6390

Éditeur :

Éditions du Centre d'études et de documentation Mémoire d'Auschwitz, Éditions Kimé

Édition imprimée

Date de publication : 2 mai 2016

Pagination : 128-135

ISSN : 2031-4183

Référence électronique

Luc Rasson, «Enric Marco, c'est moi : El impostor de Javier Cercas », Témoigner. Entre histoire et mémoire [En ligne], 122 | 2016, mis en ligne le 30 septembre 2021, consulté le 02 février 2022. URL http://journals.openedition.org/temoigner/4414 ; DOI : https://doi.org/10.4000/temoigner.4414 


\section{Enric Marco, c'est moi :} El impostor de Javier Cercas

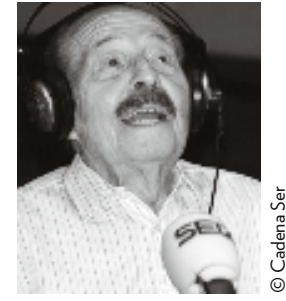

- Enric Marco $\rightarrow$ Par Luc Rasson

Université d'Anvers
(1) Voir entre autres les articles suivants : Estrella Israel Garzón et Marilda Azulay Tapiero, "Le cas Enric Marco dans l'espace public. Réactions et opinions médiatiques à propos d'un faux déporté », Témoigner entre histoire et mémoire, $\mathrm{n}^{\circ} 106$, janvier-mars 2010 p. 60; Matthew Russell, "The Holocaust as Trope in Post-Franco Spain. Historical Memory and the Case of Enric Marco », Vanderbilt e-Journal of Luso-Hispanic Studies, $n^{\circ} 6$, 2010, http://ejournals.library. vanderbilt.edu/ojs/index. php/lusohispanic/article/ view/3255/1470 (consulté le 29 avril 2015).

\begin{abstract}
« Sçachons gré au sort de nous avoir fait vivre en un siecle non mol, languissant ny oisif : tel, qui ne l'eut esté par autre moyen, se rendra fameux par son malheur.»

Montaigne, «De la Physionomie».
\end{abstract}

e cas de Enric Marco est désormais bien connu ${ }^{1}$. On se souvient de ce Catalan qui se présenta comme un ancien détenu du camp de Flossenbürg, qui fut pendant des années président de l'Amicale espagnole de Mauthausen et qui donna, en cette qualité, des centaines de conférences et d'interviews où il parla de son expérience personnelle de l'univers concentrationnaire nazi. Il connut son heure de gloire le 28 janvier 2005 lorsqu'il prononça un discours émotionnel au Parlement espagnol à l'occasion du Jour de la mémoire de la Shoah. Peu après, il fut démasqué par l'historien Benito Bermejo et contraint d'avouer qu'il avait bien été en Allemagne pendant la guerre, mais en tant que travailleur volontaire. Et s'il fut emprisonné, c'était à la prison de Kiel et non pas dans un camp de concentration. Ainsi Enric Marco s'inscrit dans la série des imposteurs se prévalant d'une expérience de déportation qu'ils n'ont jamais connue - pensons à Jerzy Kosinski, Benjamin Wilkomirski, Micha Defonseca et d'autres. Inutile de souligner l'ampleur du scandale : comment ose-t-on se glisser frauduleusement dans la peau d'un survivant, comment peut-on en arriver à usurper cette place prestigieuse ?

Mais à ces questions s'en ajoute une autre, plus inquiétante peut-être : comment le public a-t-il pu se laisser berner par un tel personnage? Comment les journalistes, les enseignants, les hommes politiques, les historiens mêmes ont-ils pu 
ajouter foi aux récits d'un usurpateur sans que personne ne s'avise de contrôler la véracité de ses dires ? Certes, depuis que nous sommes entrés dans «l'ère du témoin», sa parole est sacrée : «Qui se dit survivant ne peut mentir. $»^{2}$ Les noms d'Auschwitz, de Flossenbürg, de Buchenwald, etc., font désormais partie de notre identité, en tant que mises en garde que le $\mathrm{XX}^{\mathrm{e}}$ siècle nous adresse afin que cela ne se produise plus. Aussi celui qui peut dire «j’y fus » suscite-t-il le respect des générations ultérieures. Mais on mesure aussi la séduction qu'ont pu exercer ces noms sur les mythomanes en quête de notoriété, instrumentalisant le prestige attaché à la figure de la victime.

Les réactions à la découverte de l'imposture de Enric Marco suivirent trois lignes argumentatives ${ }^{3}$ : l'indignation inspirée par l'appropriation de la souffrance d'autrui et la crainte de l'usage que pourraient faire (et qu'ont fait) de l'imposture les négationnistes ; le constat, ensuite, que l'usurpation était mise au service d'une bonne cause - celle du devoir de mémoire - ; et enfin les opinions exprimant une forme d'admiration pour la puissance narrative et théâtrale dont fit preuve le personnage. Parmi ces dernières réactions, je m'attarde un instant sur celle de l'écrivain Mario Vargas Llosa qui au-delà de sa «répugnance morale et politique envers le personnage» avoue dans les colonnes de El País ${ }^{4}$ son «admiration de romancier pour sa prodigieuse habileté

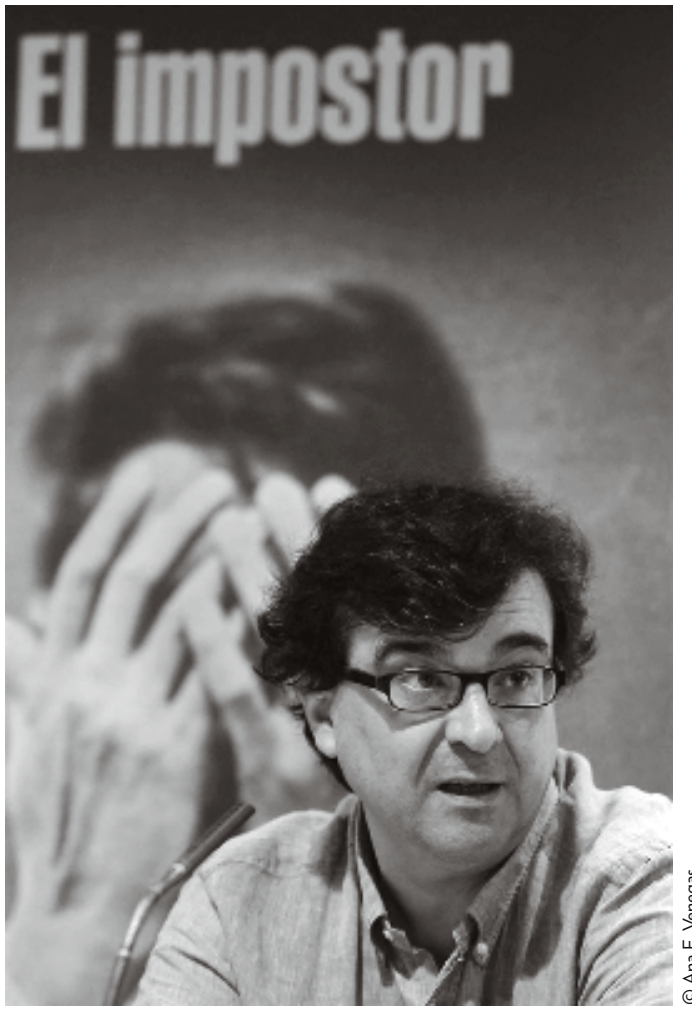

_ Javier Cercas

fabulatrice.» Admiration qui le pousse à placer Enric Marco au niveau des plus grands romanciers, de ceux qui «ont inventé et rédigé l'histoire du Quichotte, de Moby Dick, des Frères Karamazov.» Sous la plume de l'écrivain péruvien, l'usurpateur «génial» devient ce héros qui a «les pieds fermement posés dans les deux domaines» (de la littérature et de l'histoire) et qui, «[c] omme dans les meilleurs romans s'est arrangé pour les fondre inextricablement dans son existence.» Enric Marco en tant que romancier de soi-même, en tant qu'artiste ayant réussi à faire de sa vie une œuvre d'art : voilà en substance la lecture quelque peu déconcertante que propose Vargas Llosa du cas Enric Marco.

L'écrivain péruvien n'est pas le seul à être fasciné par l'aura romanesque qui entoure la figure de Enric Marco. En novembre 2014 le romancier espagnol Javier Cercas publie un roman consacré au cas qui nous occupe, El impostor ${ }^{5}$. Ce n'est pas la première fois que Cercas s'intéresse à l'Espagne du XX $\mathrm{X}^{\mathrm{e}}$ siècle et à ses récupérations mémorielles. En 2001, il publie son premier grand succès international, Soldados de Salamina où le narrateur-auteur enquête sur la figure de Rafael Sánchez Mazas, un dirigeant phalangiste proche de José Antonio Primo de Rivera, qui faillit être fusillé
(2) Estrella Israel Garzón et Marilda Azulay Tapiero, op.cit., p. 60.

(3) Ibid., p. 64.

(4) Mario Vargas Llosa, «Espantoso y genial », El País, 15 mai 2005. http://elpais. com/diario/2005/05/15/ html (consulté le 29 avril 2015, traduction de l'auteur).

(5) Javier Cercas, El impostor, Barcelone, Literatura Random House, 2014. Je continue à référer à cette édition en traduisant les passages cités.

Version française publiée depuis : L'imposteur, Élisabeth Beyer et Aleksandar Grujicic (traduction), Paris, Actes Sud , septembre 2015. opinion/1116108006_850215. 
(6) Javier Cercas, Anatomia de un instante, Barcelone, Random House Mondadori, 2010 (2009), p. 14-15.

(7) « como quien despoja de su piel a una cebolla» (p. 329). La métaphore est-elle un clin d'œil à l'autobiographie de Günther Grass, Pelures d'oignon (2006), où l'écrivain allemand avoua avoir fait partie de la SS? par un soldat républicain à la fin de la guerre civile. Dans Anatomía de un instante, publié en 2009, le romancier raconte le coup d'État raté du 23 février 1981 en se focalisant sur les trois «héros du renoncement» qui tinrent tête aux insurgés et qui permirent de la sorte la sortie définitive de l'Espagne de l'ère de la dictature : le Premier ministre Adolfo Suárez, le chef du parti communiste Santiago Carrillo et le général ex-franquiste Gutiérrez Mellado. Avec El impostor, Javier Cercas poursuit sa réflexion sur la mémoire du $\mathrm{XX}^{\mathrm{e}}$ siècle espagnol en s'intéressant à Enric Marco. Mais le lien entre ces trois romans n'est pas que thématique : il est aussi formel, et cela tout particulièrement entre Anatomía de un instante et El impostor, où le narrateur-romancier part du constat que les événements historiques en question sont déjà saturés de fiction. C'est le cas pour le coup d'État du 23 février qui est probablement le seul à avoir été entièrement filmé en direct - or, avance le romancier, «la télévision contamine d'irréalité tout ce qu'elle touche», ce qui permet de conclure que «dans la société du spectacle, le coup d'État fut un spectacle de plus. ${ }^{6}$ De même, on le sait, l'histoire d'Enric Marco est déjà une fiction.

À quoi bon dès lors faire de la fiction sur une fiction? À quoi bon écrire un roman sur une personne qui a fait de la fiction la raison d'être de sa vie ? À une première approche, le problème que pose le cas Enric Marco appelle une réponse éthique. Voilà quelqu'un qui a trompé sciemment les gens, qui a usurpé la place de la victime et cela, pour reprendre l'expression récurrente de Javier Cercas, afin de «paraître sur la photo » - d'être au centre de l'attention et de récolter les louanges de la part de ceux qui se sont fait abuser. La réponse esthétique en revanche - l'écriture d'un roman par exemple - paraît moins évidente, du moins à première vue. La réalité même de l'affaire ne parle-t-elle pas assez pour elle-même? Le scandale a-t-il besoin de se dire en termes romanesques pour éclater aux yeux ? L'intelligence du roman de Javier Cercas réside dans le fait que toutes ces questions, le narrateur se les pose à longueur de pages, dans une réflexion métacritique incessante qui l'amène à réexaminer le genre romanesque. Car si traditionnellement le roman se propose comme ce détour par la fiction qui permet de mieux saisir la réalité - ou de mettre à nu ses non-dits - le roman tel que le conçoit Cercas se propose en revanche de gratter les couches de fiction que contient le passé du personnage, «comme celui qui enlève la peau d'un oignon. $»^{7}$ Face à l'imposteur, la tâche du romancier ne saurait être d'ajouter de la fiction à la fiction : il s'agira, au contraire, de rétablir la réalité dans ses droits. Voilà ce que Javier Cercas appelle la «novela sin ficción » ou roman sans fiction. Si le projet peut paraître paradoxal, on notera toutefois que l'écrivain demeure ainsi fidèle à la vocation critique du roman qui consiste à décrédibiliser les discours perçus comme étant inauthentiques. Débusquer le mensonge, démystifier l'aura d'un narrateur, dénoncer le caractère faussement naturel d'une prise de parole et éventuellement, au bout de tout cela, rétablir une vérité occultée ou travestie, voilà quelques-unes des tâches que le roman, au moins depuis le Don Quichotte, s'est assignées. Et c'est très exactement ce que fait Javier Cercas, non sans quelques ambiguïtés que j’aimerais pointer ici. 


\section{MALAISES}

Voici le défi posé au romancier : comment représenter une parole qui n’offre aucune garantie d'authenticité alors qu'elle est investie du prestige majeur de la victime et du témoin? Enric Marco a bâti sa vie sur un mensonge, à savoir qu'il est un ancien de Flossenbürg. Comment peut-on dès lors le prendre au sérieux dans le reste de ses affirmations? C'est bien connu: qui a la réputation d'être un menteur ne sera plus jamais pris au sérieux - même quand il dit la vérité. Le personnage invite donc à une suspicion incessante. Tout ce qu'il raconte avec la force du témoin n'est peut-être qu'invention. Aussi cette prise de parole entraîne-t-elle la mise en place d'un certain nombre de procédés permettant de la canaliser, de la contenir, de la contredire. Discrédit jeté sur le personnage, pour commencer. La première visite du romancier à l'imposteur se passe mal. Au dégoût physique - «il me paraissait être une espèce de gnome » - s'ajoute la réticence qu'inspire le «hâbleur compulsif » (p.78) incapable ou peu enclin à cacher son narcissisme: en effet, Marco n'arrête pas de parler de lui-même. Sortant de chez l'imposteur, le narrateur conclut à l'impossibilité d'écrire sur ce «monstre de vanité et d'égotisme» (p. 41). La deuxième façon d'exprimer le soupçon est de nature discursive. Lorsque Marco parle, le narrateur représente son discours sur le mode indirect, ne le citant donc pas littéralement, mais en multipliant les verbes déclaratifs : «il dit que», «il raconte que», «il se souvient de...» - comme pour bien signifier au lecteur que cette parole, équivoque par définition, ne peut pas être prise pour argent comptant, et qu'il faut mettre en œuvre une vigilance de tous les instants afin de bien distinguer la parole du narrateur de celle du faux témoin : il s'agit d'éviter à tout prix l'amalgame. Enfin le narrateur intègre dans son récit - troisième procédé - la critique explicite du discours d'Enric Marco. Ainsi les chapitres où le personnage raconte un épisode de sa vie sont suivis par des chapitres où le narrateur décortique le discours de l'usurpateur, le soumet à une critique historique soutenue, afin de tenter d'en dégager le degré de vérité.

Cela dit, la représentation doublée de la critique du discours du narrateur nonfiable ${ }^{8}$ qu'est Enric Marco ne va pas de soi. Elle pose des problèmes de nature littéraire, historique et morale. Pour commencer, le narrateur-romancier nous l'assène d'entrée de jeu, Enric Marco n'est pas exclusivement l'autre. En effet, lors d'un dîner chez Mario Vargas Llosa, le narrateur se fait traiter lui aussi d'imposteur. Le romancier qui invente des mondes fictifs en les présentant comme étant authentiques, ne l'est-il pas par définition ? En quoi le recours à la fiction fonderait-il l'autorité du romancier alors qu'il jetterait le discrédit sur la prise de parole d'Enric Marco qui est lui aussi pourvu d'une «imagination romanesque» (p. 85) ? Par ailleurs, la dimension performative du discours du faux déporté - presque tout le monde y a cru - n'est-elle pas l'indice du fait que, comme le romancier, il tire son pouvoir des mots ? Certes, il y a une différence : le romancier a le droit de mentir et le lecteur, selon la formule consacrée, suspend volontairement son incrédulité alors qu'Enric Marco a sciemment trompé un public qui, étant donné l'autorité accordée d'avance
(8) Ou « unreliable narrator », selon la définition canonique de Wayne Booth : «I have called a narrator reliable when he speaks for or acts in accordance with the norms of the work (which is to say the implied author's norms), unreliable when he does not ».

The Rhetoric of Fiction, Chicago et Londres, University of Chicago Press, 1969 [1961 ], p. 158-159. 
à la parole du témoin, ne demandait qu’à croire. La question que pose le cas d'Enric Marco concerne donc la littérature, et elle n'est pas étrangère aux préoccupations du romancier même. D’ailleurs le roman n'a-t-il pas toujours été fasciné par des personnages vivant dans et par la fiction - et cela depuis au moins le Don Quichotte?

Une deuxième question qui révèle le malaise du narrateur est classique dans le cadre des «Perpetrator studies » : est-ce que comprendre le bourreau équivaut à le justifier ? Question posée déjà par Primo Levi, sans réponse concluante, car le narrateur ne manque pas de pointer la contradiction que l'auteur de Si c'est un homme n'a pas dépassée, à savoir de poser l'équivalence entre comprendre et justifier. Assurément, Enric Marco n'est pas un bourreau, mais il soulève les mêmes interrogations, car vouloir comprendre l'usurpateur - qui, d'une certaine façon, a intensifié la souffrance des vraies victimes - vous oblige à vous mettre dans sa peau, $\grave{a}$ faire sonjeu. Est-ce que le narrateur, en voulant savoir, ne se fait pas le complice de l'imposteur? Que ces questions sont particulièrement délicates ressort par exemple des polémiques qu'ont déclenchées des romans essayant de cerner la figure du bourreau en racontant les choses de leur point de vue ou avec leurs mots : c'est le cas, entre autres, de La mort est mon métier (1952) de Robert Merle ou des Bienveillantes (2006) de Jonathan Littell. Or, je l’ai déjà signalé, Enric Marco parle souvent par la bouche du narrateur : il y a dans El impostor des phénomènes d'hybridité narrative qui introduisent un brouillage énonciatif quand bien même le narrateur prend soin, dans la mesure du possible, de distinguer la voix de l'usurpateur de la sienne.

Enfin, le narrateur se demande s'il a le droit d'écrire sur Enric Marco. En effet, le personnage de l'imposteur est encore en vie et la publication d'un roman qui lui est consacré pourrait avoir des conséquences pour lui-même, pour sa famille et ses proches. Le problème, rappelle le narrateur, s'est posé aussi à Emmanuel Carrère dans L'adversaire, quand il s'est intéresséà un autre imposteur, Jean-Claude Romand, ainsi qu’à Truman Capote confronté aux jeunes assassins décrits dans De sang froid. Dans tous ces cas, le fait de vouloir tirer un roman de ces personnages infâmes oblige le romancier à faire des compromissions où sa propre intégrité morale est mise en cause : Truman Capote était conscient du fait que la condamnation à mort des deux jeunes malfrats serait la meilleure conclusion à son livre et Javier Cercas, pour sa part, savait que son imposteur «médiopathe», désireux tout au long d'être sur l'avant-plan serait ravi d'être le protagoniste d'un roman, quand bien même il y jouerait le méchant rôle : il est des gens qui jouissent de la publicité qu’on leur fait même quand elle est négative.

\section{LE KITSCH HISTORIQUE}

Enric Marco révèle donc un malaise. Un malaise qui paralyse le romancier, car ce roman est aussi l'histoire de l'écriture du roman, des doutes qui assaillent l'écrivain et de la réticence à écrire. Un «livre impossible», selon le narrateur, ou 
encore, une «noble défaite» qui serait «le maximum à quoi peut aspirer un écrivain.» (p. 54) Et pourtant le roman est là, sous les yeux du lecteur, pour son plus grand plaisir. C'est que, au-delà des tergiversations du narrateur-romancier, le cas Enric Marco a une valeur de symptôme. En effet, sans le vouloir l'imposteur révèle quelque chose sur l'état de la «mémoire historique», ainsi que sur la littérature et cela surtout en Espagne où la notion de la fiction empiétant sur le réel a une résonance culturelle particulière.

Le faux témoignage de Enric Marco est le succédané d'un discours authentique. En tant que tel il relève du kitsch - défini comme un discours ou une pratique qui occulte la réalité sous une façade de sentimentalisme et de fausse vertu. Lorsque le protagoniste raconte comment, au mépris de sa vie, il a battu un officier SS aux échecs, il propose une vision mélodramatique de l'expérience concentrationnaire dans laquelle il se réserve le beau rôle du héros en carton-pâte qui ose se dresser symboliquement contre la bête nazie. Récit tout entier axé sur l'effet qu'il est censé produire sur son auditoire, mais qui évacue toutes les contradictions et les ambiguïtés de la situation historique. Or, en tant que tel le témoignage de Enric Marco n'est pas isolé. Il est représentatif de ce que le romancier appelle la «nouvelle industrie de la mémoire» (p. 188) qui, selon lui, aurait pris le dessus en Espagne dans la première décennie du XXI ${ }^{\mathrm{e}}$ siècle, au moment où l'on a commencé à prendre en compte la mémoire des victimes de la guerre civile. Marco serait donc «l'emblème» de l’industrie de la mémoire, « une industrie qui fonctionne à plein rendement non seulement en Espagne, mais aussi en Europe et aux États-Unis.» ${ }^{9}$ Et ce constat permet au romancier de critiquer la Loi de la mémoire historique de 2007 qui prétendait rétablir dans leurs droits les victimes de la guerre civile et de la dictature. Selon Javier Cercas, cette loi est restée lettre morte précisément parce que la nécessité historique s'est muée en une mode qui a donné lieu à la commercialisation - à ce kitsch que Enric Marco a contribué à répandre, à l'image de tant d'autres Espagnols qui ont réinterprété leur passé et se sont forgés après coup une carrière d'opposants.

En d'autres mots, Enric Marco n'est pas l'exception, il est la règle - du moins dans l'Espagne de la transition vers la démocratie ${ }^{10}$. Or, ce n'est peut-être pas tout à fait un hasard si le personnage appartient à la sphère culturelle hispanique. Il faut parler ici de la prédilection espagnole pour le thème du brouillage entre réalité et fiction dont le Don Quichotte est évidemment la première grande expression, mais qui a continué à fasciner les écrivains de la péninsule tels Benito Pérez-Galdós ou Miguel de Unamuno et, au-delà, ceux de l'aire hispanique élargie, tels Jorge Luis Borges ou Julio Cortázar. Or, tout au long du roman, le personnage de l'usurpateur est systé-

(9) Daniel Gascon, «Entrevista con Javier Cercas. Toda gran literatura es literatura comprometida». http://www.letraslibres.com/ revista/entrevista/entrevistacon-javier-cercas (consulté le 6 mai 2015). Je remercie Jasper Vervaeke de mavoir signalé cette interview.

(10) «y de ahí que, en aquella época de reinvenciones masivas, Marco no fuera una excepción sino la regla » (p. 299). 
(11) Cervantès, L'Ingénieux Hidalgo Don Quichotte de la Manche, Deuxième partie, Paris, Gallimard, 1949. Folio n ${ }^{\circ} 1901$, p. 601. (Traduction de François de Rosset revue par Jean Cassou).

(12) «Marco había construido a lo largo de casi un siglo la mentira monumental de su vida (...) para que un escritor futuro la descifrase con su ayuda y luego la diese a conocer por el mundo, igual que Alonso Quijano había construido a Don Quijote (...) para que Cervantes las descifrase» (p. 408). matiquement indexé au héros de Cervantès. Le lecteur l'apprend dès les premières pages, lorsqu'un personnage affirme : «Enric est égal à Don Quichotte. Il n’a pas pu se contenter de vivre une vie médiocre, il a voulu vivre une vie prestigieuse; comme elle était hors d'atteinte, il se l'est inventée.» (p. 33) Plus fort encore que le chevalier de la triste figure, le Catalan est un imposteur qui a réussi son coup - jusqu'au moment, du moins, où intervient l'historien Benito Bermejo. En effet Alonso Quijano, qui a cinquante ans commence à se prendre pour un chevalier errant, n’abuse personne : son entourage sait très bien qu'il a un grain. Enric Marco en revanche a persuadé tout le monde qu'il était un ancien de Flossenbürg.

Il ne fait pas de doute que la comparaison du faux témoin des camps avec le personnage de Don Quichotte peut jeter une lumière intéressante sur le cas Enric Marco. Cependant, le rapprochement génère quelques effets ambigus. Il souligne, avant tout, l'ambivalence des rapports qui relient le romancier et l'imposteur, car le romancier n'est-il pas toujours complice de son personnage («Madame Bovary, c'est moi»)? C'est du moins ce que suggère le narrateur lui-même dans une réflexion - une de plus - sur Cervantès. À la fin du Don Quichotte, la parole est donnée à la plume de l'écrivain, qui affirme : «Pour moi seul naquit Don Quichotte, et moi pour lui. Il sut agir, moi écrire. Enfin, lui et moi ne sommes qu'une même chose. ${ }^{11}$ La plume de l'écrivain et son protagoniste sont les deux faces d'une seule instance et Javier Cercas prend un malin plaisir à paraphraser la phrase de son illustre prédécesseur : «Pour moi seul naquit Enric Marco, et moi pour lui. Il sut agir, moi écrire. Enfin, lui et moi ne sommes qu'une même chose.» (p. 333) Qu'est-ce qui est suggéré ici ? Que l'imposture du personnage ne fut, au bout du compte, que le prétexte à l'écriture d'un beau roman? De la sorte El impostor serait la récupération esthétique d'une histoire qui pourtant interpelle d'abord par ses implications éthiques. Or, si Enric Marco n'est que cela - l'occasion d'une réussite littéraire - ne faut-il pas se demander en dernière instance qui se joue de qui ? Peut-être, suggère le narrateur, Marco a-til construit son leurre afin qu'un écrivain en fasse son miel - de même que Alonso Quijano a inventé Don Quichotte afin que Cervantès s'en empare ${ }^{12}$...

Il y a plus. La mise en équivalence constante de Enric Marco avec le protecteur des veuves et des orphelins tend à élever le personnage à un statut prestigieux, quasi mythique - non pas, comme il l'avait escompté, de survivant des camps, mais de personnage littéraire devenu un archétype universel. De la sorte le scandale mémoriel se voit désarçonné et le personnage d'Enric Marco amalgamé aux grands personnages non seulement de la littérature, mais aussi de la mythologie, puisque Cercas développe également les analogies avec les figures d'Icare et de Narcisse. Et le lecteur arrivé au bout du roman se demande si, tout compte fait, Enric Marco qui a aujourd'hui 94 ans et dont on connaît la soif de publicité, n'est pas ravi de l'hommage intelligent que lui rend, avec El impostor, un des grands romanciers espagnols d'aujourd'hui - hommage qui ne manquera pas d'assurer sa survie posthume. 


\section{MARCO, C'EST NOUS}

Dans un long dialogue imaginaire entre l'usurpateur et le romancier, le premier lance, non sans effronterie, la question suivante à la face du narrateur : «Connaissez-vous un thème plus passionnant que moi ?» (p. 355) Question qui résume bien les ambiguïtés cernées par ce roman. Car, cela ne fait aucun doute, Enric Marco fascine. Au-delà de la réaction première de l'indignation, on ne peut s'empêcher de s'intéresser à ce personnage qui a fait de sa vie un roman et qui a réussi, en outre, à le rendre crédible. Mais le paradoxe que désigne Javier Cercas réside dans le fait que Enric Marco n'est pas, on l'a vu, un cas exceptionnel. Il n'est pas le seul Espagnol de la transition à avoir embelli sa biographie. Marco, signale le narrateur pour finir, a toujours été un homme de son époque. Républicain quand tout le monde l'était dans le Barcelone de la guerre civile ; citoyen obéissant et docile sous la dictature franquiste, ne se risquant jamais à faire de l'opposition ; individu libéré après la mort du dictateur, se réinventant un passé glorieux de militant de gauche. Enric Marco s'est toujours situé là où se trouvait la majorité. Aussi « l'énigme » est-elle, au bout du compte, «son absolue normalité» (p. 412). Sommes-nous bien sûrs d'être différents? Enric Marco est ce qui reste quand toutes les pelures de l'oignon ont été enlevées, c'est-à-dire rien, ce «point aveugle» (p. 412) à partir duquel la critique de certains usages contemporains de la mémoire devient possible. Car ce roman est avant tout une mise en garde contre la mémoire gelée, émaillée de lieux communs mélodramatiques et de topoï épiques dont le seul bénéfice est l'autopromotion du sujet exhibant sa bonne conscience : écoutez-moi, bonnes gens, voyez comme j'incarne la vertu historique - après coup. Posture qui ne se limite pas au seul usurpateur. I 